\title{
Que peuvent nous révéler les données des ambulanciers paramédicaux sur la crise des opioïdes au Canada?
}

\author{
Minh T. Do, Ph. D. (1, 2, 3); Greg Furlong, ACP (4); Micah Rietschlin, PCP (4); Matthew Leyenaar, M.A. (5); \\ Michael Nolan (6); Pierre Poirier, M.B.A. (7); Brian Field, M.B.A. (8); Wendy Thompson, M. Sc. (1)
}

Diffuser cet article sur Twitter

\section{Résumé}

La nature de la crise des opioïdes au Canada nécessite des sources de données supplémentaires aptes à dresser un portrait plus fidèle de l'épidémie, afin de fournir aux responsables en santé publique et aux décideurs une base de données probantes solide. Les données des ambulanciers paramédicaux sont un point d'accès aux collectivités où les surdoses surviennent.

Les événements préhospitaliers et les circonstances entourant les surdoses d'opioïdes offrent des occasions uniques de recueillir des données probantes pouvant contribuer à la prévention, à la réduction des méfaits et aux efforts de promotion de la santé. À l'aide de données extraites du Service paramédic d'Ottawa (SPO), cette étude de validation de principe a démontré que les données d'intervention ambulancière paramédicale étaient utiles pour obtenir des renseignements épidémiologiques en temps quasi réel (personne, heure et lieu) sur l'épidémie d'opioïdes et pour évaluer les tendances ainsi que les possibilités d'élaborer des déclencheurs d'alerte.

Entre janvier et juin 2017, le SPO a répondu à une moyenne de quatre appels liés aux opioïdes par semaine. À chaque fois, $0,5 \mathrm{mg}$ de naloxone ont en moyenne été administrés. Pour la période à l'étude, les tendances linéaires montrent une faible augmentation des appels, non significative ( $\mathrm{p}=0,18)$. Le volume d'appels a augmenté entre le 16 et le 29 avril 2017. Selon les médias locaux, ce pic dans les interventions ambulancières paramédicales est attribuable à l'arrivée de fentanyl de qualité supérieure à Ottawa.

Avec une validation plus poussée, ces données paramédicales pourraient potentiellement constituer une nouvelle source de données pour la surveillance des surdoses liées aux opioïdes.

Mots-clés : opioïdes, ambulancier paramédical, soins préhospitaliers, système d'alerte rapide, Canada

\section{Introduction}

Le Canada est au cœur d'une épidémie d'opioïdes. Dans plusieurs régions du pays, le nombre de décès est à la hausse ${ }^{1}$, avec peu de signes d'atténuation de la crise. En 2016, 2861 décès ont été attribués à l'usage apparent d'opioïdes ${ }^{1}$ et, si la tendance actuelle se maintient, ce total devrait s'élever à plus de 4000 en $2017^{1}$.

En réaction, diverses ressources ont été mobilisées afin de surveiller l'épidémie de près. Par exemple, grâce à la collaboration

\section{Points saillants}

- Les données paramédicales peuvent fournir des renseignements sur les soins préhospitaliers utilisables pour la surveillance et la détection systématiques des changements soudains dans le nombre d'événements liés aux opioïdes dans la collectivité.

- Certaines des personnes ayant fait une surdose n'étant pas transportées à l'hôpital, leur dossier paramédical peut constituer le seul enregistrement médical de leur surdose.

- Dans cette étude de validation de principe, des pics importants dans le nombre d'interventions des ambulanciers paramédicaux lors d'événements liés aux opioïdes ont été détectés avant que les médias ne rapportent l'arrivée de fentanyl de qualité supérieure à Ottawa (Canada).

entre les provinces et les territoires, les délais de consignation des données sur les décès liés aux opioïdes ont été considérablement réduits. De même, une surveillance accrue des visites aux services d'urgences a également été mise en place. En Ontario, les hôpitaux sont tenus de déclarer sur une base hebdomadaire les visites à l'urgence liées aux opioïdes² . Toutefois, malgré une intervention coordonnée en santé publique, il demeure toujours des lacunes en matière de données pour la surveillance systématique des

Rattachement des auteurs :

1. Agence de la santé publique du Canada, Ottawa (Ontario), Canada

2. École de santé publique Dalla Lana, Université de Toronto, Toronto (Ontario), Canada

3. Faculté des sciences de la santé, Université Carleton, Ottawa (Ontario), Canada

4. Service paramédic d'Ottawa, Ottawa (Ontario), Canada

5. Université McMaster, Hamilton (Ontario), Canada

6. Renfrew County Paramedic Service, Renfrew (Ontario), Canada

7. Association des paramédics du Canada, Ottawa (Ontario), Canada

8. Interdev Inc., Toronto (Ontario), Canada

Correspondance : Minh T. Do, Division de la surveillance et de l'épidémiologie, Agence de la santé publique du Canada, 785, avenue Carling, Ottawa (Ontario) K1A 0K9; tél. : 613-797-7587; téléc. : 613-941-2057; courriel : minht.do@canada.ca 
surdoses dans les collectivités de l'ensemble du pays.

Au Canada, les ambulanciers sont habituellement les premiers professionnels de la santé à arriver sur les lieux d'une surdose et ils ont l'habitude de recueillir des données sur ses circonstances. Dans la plupart des administrations, ces données " préhospitalières " sont saisies dans un système électronique dans les 24 heures suivant l'événement. Ces renseignements peuvent se révéler d'une valeur inestimable pour comprendre le contexte de la crise des opioïdes. Dans cette optique, l'objectif de cette étude de validation de principe était de déterminer si les données paramédicales peuvent être utilisées couramment à des fins de surveillance en santé publique.

\section{Méthodologie}

Le Service paramédic d'Ottawa (SPO) recueille des données sur la répartition et les urgences médicales dans la ville d'Ottawa (qui compte environ un million d'habitants sur une superficie de 2796 kilomètres carrés). Il recueille de l'information sur les caractéristiques des patients, la nature de l'intervention d'urgence et le traitement s'il y a lieu, par exemple la dose de naloxone administrée.

Pour cette étude de validation de principe, nous avons extrait le 27 juin 2017 de l'OPS les données recueillies entre le $1^{\mathrm{er}}$ janvier et le 17 juin 2017. Bien que les données de géocodage aient été disponibles (emplacement de la surdose par adresse et codes postaux complets), ces données n'ont pas été affichées pour des raisons de protection de la vie privée.

\section{Méthodes statistiques}

Nous avons utilisé des données anonymes agrégées pour cette analyse. Un événement lié aux opioïdes a été défini comme une intervention nécessitant l'administration de naloxone pour contrecarrer les effets d'une surdose liée aux opioïdes. En raison du petit nombre de cas, les événements ont été rapportés sur une base hebdomadaire et agrégés par tranche d'âge de cinq ans. Nous avons produit des statistiques descriptives pour examiner la répartition des événements dans le temps.

Pour l'analyse des tendances, nous avons établi un seuil d'alerte. Nous avons utilisé une moyenne mobile de 7 semaines pour calculer des valeurs prévues stables. Compte tenu de la nature quantitative des données, nous avons utilisé la distribution de Poisson pour associer un intervalle de confiance (IC) à $95 \%$ à la valeur prévue ${ }^{3}$. Nous avons utilisé des seuils statistiques $(\alpha=0,05)$ de façon arbitraire pour définir les seuils d'alerte. Nous avons utilisé pour nos analyses un gabarit Excel avec macros produit par le European Centre for Disease Prevention and Control (ECDC) ${ }^{3}$. Nous avons considéré comme un événement seuil ou un écart par rapport à la fourchette prévue un nombre d'interventions paramédicales dépassant les limites supérieures fixées et nous lui accordé un examen plus approfondi (niveau d’alerte).

\section{Résultats}

Entre le $1^{\text {er }}$ janvier 2017 et le 17 juin 2017, 86 interventions des ambulanciers sur les lieux d'une surdose liée aux opiö̈des ont nécessité l'administration de naloxone. À chaque fois, une dose moyenne de $0,5 \mathrm{mg}$ de naloxone a été administrée. Les deux tiers des cas (66\%) concernaient des hommes et, dans $57 \%$ de l'ensemble des cas, les victimes avaient entre 25 et 39 ans (mode : 30 à 34 ans).

Les tendances linéaires montrent une augmentation faible mais non significative du nombre de réponses hebdomadaires des ambulanciers $(\mathrm{p}=0,18)$. Au cours de la période à l'étude, le SPO a reçu une moyenne de quatre appels par semaine. Entre le 16 et le 29 avril, le nombre d'interventions a dépassé les seuils (figure 1). Le plus grand nombre d'appels a été reçu au cours de la semaine 17 (du 23 au 29 avril 2017), avec 9 appels ayant donné lieu à l'administration de naloxone par des ambulanciers. La hausse du nombre d'appels a été signalée dans la presse locale et les médias électroniques ${ }^{4,5}$, puis dans les médias sociaux une semaine plus tard ${ }^{6}$.

\section{Analyse}

Le but de cette étude était d'évaluer si les données sur les interventions paramédicales étaient utilisables pour la surveillance de la crise des opioïdes. La surveillance en santé publique peut être définie comme [traduction] «la collecte, l'analyse et l'interprétation systématiques et continues des données, en lien étroit avec la diffusion opportune et cohérente des résultats et de l'évaluation à ceux qui ont le droit de savoir afin que des mesures puissent être prises $»^{7}$. Dans ce contexte, la collecte systématique de données par le SPO a permis de fournir des données épidémiologiques sur la personne, le lieu et l'heure - renseignements nécessaires à la surveillance en santé publique.

Ces données sont cohérentes avec ce que l'on sait de la crise des opioïdes au Canada. La plupart des interventions des ambulanciers (66 \%) ont été liées à des surdoses d'opioïdes chez des hommes, ce qui correspond aux données sur les décès (74 \% des décès apparemment liés aux opioïdes sont survenus chez les hommes ${ }^{1}$ ). De même, les données sur les soins préhospitaliers (données sur les interventions paramédicales) et hospitaliers (données sur les visites à l'urgence et les admissions à l'hôpital) indiquent que les jeunes adultes courent un risque élevé de surdoses liées aux opiö̈des ${ }^{8}$. La collecte continue de données par le SPO a également permis d'évaluer les tendances, avec des pics de dépassement des seuils statistiques, avant que les médias n'en fassent état. Ces renseignements sur la santé sans délai d'exécution pourraient s'avérer inestimables pour la prise de mesures en santé publique.

Dans la plupart des régions du Canada, les données d'intervention sont entrées dans les bases de données électroniques dans les 24 heures suivant l'intervention d'urgence des ambulanciers. Les données sont ainsi rendues accessibles pour une analyse en temps quasi réel et peuvent de ce fait contribuer à un système d'alerte précoce.

Deuxièmement, les données d'intervention des ambulanciers sont géocodées (données non présentées), car les ambulanciers ont besoin de l'emplacement exact pour réagir à un événement. Des informations précises indexées géographiquement pourraient ainsi être utilisées pour la surveillance en santé publique afin d'aider à identifier les points chauds et à produire des cartes de chaleur. Troisièmement, comme les personnes qui font une surdose ne sont pas toutes transportées à l'hôpital, leur dossier paramédical pourrait constituer le seul enregistrement de leur surdose.

Enfin, les données d'intervention paramédicale seraient utilisables pour évaluer les répercussions d'une politique ou d'une intervention particulière en matière de santé publique. Par exemple, la politique consistant à mettre des trousses de naloxone à la disposition du grand public sans 
FIGURE 1

Distribution des valeurs observées et prévues et seuils d'intervalle de confiance à $95 \%$ pour le nombre d'interventions paramédicales du Service paramédic d'Ottawa pour les cas de surdoses liées aux opioïdes ayant nécessité de la naloxone, du $1^{\text {er }}$ janvier 2017 au 17 juin 2017, Ottawa, Canada

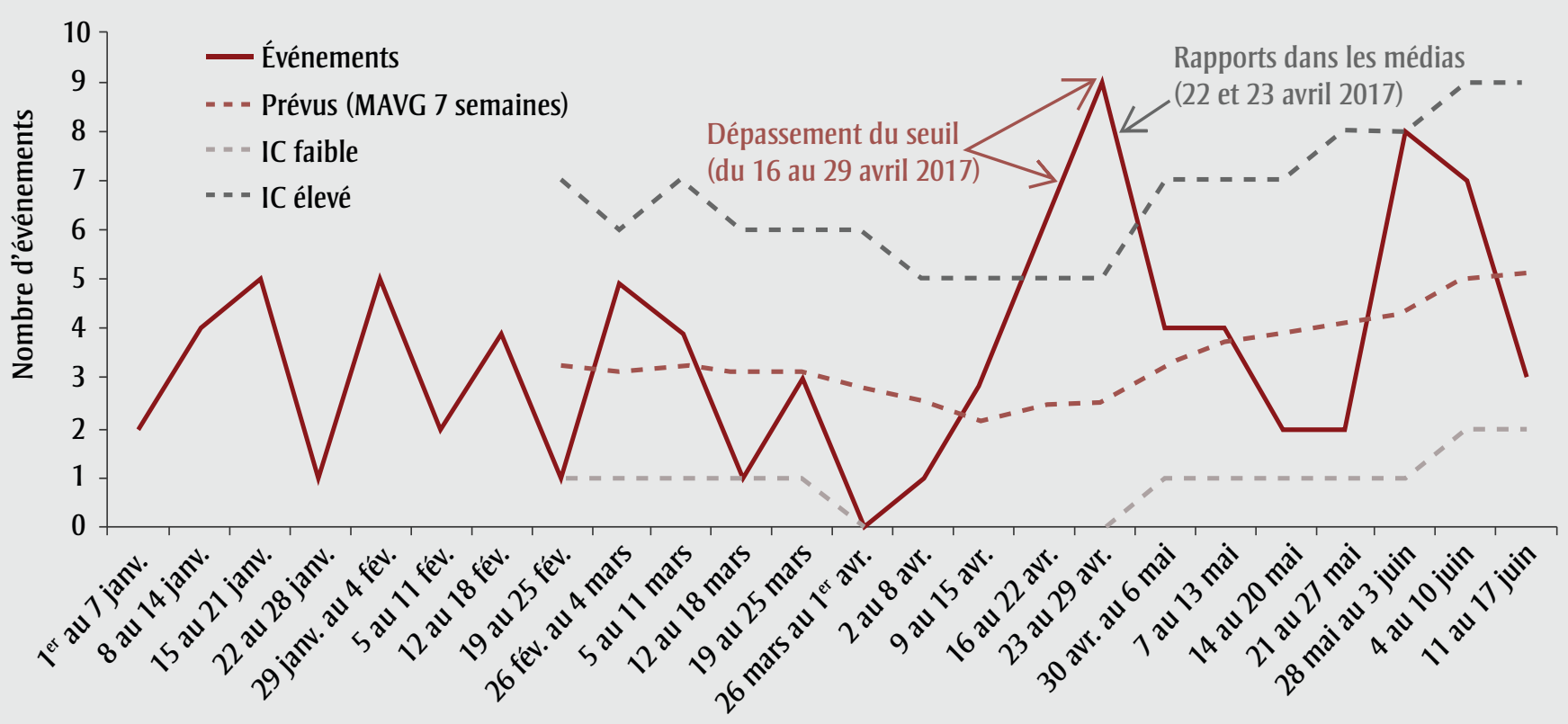

Abréviations : IC, intervalle de confiance; MAVG, moyenne mobile.

ordonnance pourrait faire en sorte que moins de personnes soient transportées aux services d'urgence, du fait que la naloxone inverse les effets de la surdose liée aux opioïdes. De plus, comme les services paramédicaux ont une vaste couverture et sont présents dans la plupart des municipalités au Canada, on pourrait brosser un tableau national de la crise des opioïdes.

Malgré ces avantages en matière de surveillance en santé publique, l'utilisation des données d'intervention paramédicale comporte des limites. Il s'agit notamment des différences dans les infrastructures, les mécanismes de fixation des priorités et les définitions de cas des surdoses liées aux opioïdes utilisées par les fournisseurs de services paramédicaux. Dans certaines administrations, les données sur les services paramédicaux sont disponibles au niveau provincial, alors que dans d'autres, elles ne le sont qu'au niveau municipal. On doit par conséquent évaluer la qualité des données pour en assurer la cohérence, l'exactitude et la précision.

D'un point de vue national, bien qu'il soit souhaitable de rassembler les données paramédicales pour obtenir un portrait d'ensemble, il est impossible de comparer les surdoses liées aux opioïdes entre les différentes administrations si leurs définitions de cas sont différentes. Il faudrait donc harmoniser les définitions de cas avant de faire des comparaisons entre administrations. Dans le cas inverse, il demeurerait utile de pouvoir se servir de l'évolution des tendances comme moyen de surveiller la crise des opioïdes. Par ailleurs, il est possible de mal classer les événements liés aux opioïdes (par exemple, les personnes qui souffrent d'une surdose liée aux opioïdes peuvent ne pas recevoir de naloxone et donc ne pas répondre à la définition de cas).

Un autre élément à prendre en considération est que les ambulanciers ne sont pas forcément appelés en cas de surdose liée aux opioïdes dans la collectivité, ce qui fait que les données de la FPO ne tiennent probablement pas compte d'une partie des surdoses.

Nous avons utilisé un seuil statistique $(\alpha=0,05)$ pour établir le seuil d'alerte. Il s'agit d'une décision arbitraire, dans le cas de notre étude qui est une étude de validation de principe. Selon le contexte, on pourrait établir un seuil statistique moins conservateur (p. ex. $\alpha=0,1$ ) ou un seuil clinique.

\section{Conclusion}

Les données d'intervention paramédicale sont aptes fournir des renseignements sur les soins préhospitaliers utilisables pour la surveillance et la détection systématiques des changements soudains dans le nombre d'événements liés aux opiö̈des au sein d'une collectivité. Dans cette étude de validation de principe, des pics importants dans les réactions des ambulanciers aux événements liés aux opioïdes à Ottawa ont été détectés avant que les médias n'en fassent état. Avec une validation plus poussée, les données d'intervention paramédicale peuvent donc potentiellement constituer une nouvelle source de données pour la surveillance des événements en santé publique tels que ceux liés aux surdoses liées aux opioïdes.

\section{Contributions des auteurs et avis}

MD a conçu, analysé et interprété les données et a rédigé et révisé le document. GF et MR ont interprété les données et rédigé et révisé le document. ML a analysé et interprété les données et révisé le document. MN, PP, BF et WT ont interprété les données et révisé le document.

Le contenu et les opinions exprimées dans cet article sont ceux des auteurs et ne reflètent pas nécessairement ceux du gouvernement du Canada. 


\section{Références}

1. Agence de la santé publique du Canada, Comité consultatif spécial sur l'épidémie de surdoses d'opioïdes. Rapport national : Décès apparemment liés à la consommation d'opiö̈des (décembre 2017) [Internet]. Ottawa (Ontario), gouvernement du Canada; 2017. En ligne à : https://www.canada .ca/fr/sante-publique/services /publications/vie-saine/deces -apparemment-lies-consommation -opioides-rapport-2016-2017-decembre .html

2. Institut canadien d'information sur la santé (ICIS). NACRS ED reporting for opioid overdose. Bulletin. Ottawa (Ontario) : ICIS; 2017. En ligne à : https://www.oha.com/Bulletins/NACRS \% 20ED \% 200pioid \% 20Reporting \% 20 Bulletin_Feb2017.pdf

3. European Centre for Disease Prevention and Control (ECDC). Handbook on implementing syndromic surveillance in migrant reception/detention centres and other refugee settings. Stockholm (SE) : ECDC; 2016.

4. CBC News. Spike in Ottawa drug overdoses continues through weekend [Internet]. Canadian Broadcasting Corporation. 2017, 24 avril [consultation le 15 février 2018]. En ligne à : www .cbc.ca/news/canada/ottawa/drug -overdose-ottawa-hospital-1.4082752

5. Duffy A. Surge in overdoses feared as more fentanyl arrives in Ottawa [Internet]. Ottawa Citizen. 2017, 23 avril [consultation le 15 février 2018]. En ligne à : http://ottawacitizen.com/news /local-news/six-more-opioid-overdose -victims-treated-at-the-ottawa-hospital

6. @DUAL Ottawa. High grade fentanyl influx in Ottawa people PLEASE be safe!!! And carry Nalozone!!! 2017, 21 avril. En ligne à : https://twitter.com/ DUALOttawa?ref_src $=$ twsrc $\% 5 \mathrm{Etfw}$ \&ref_url $=$ http $\% 3$ A $\% 2$ F $\% 2$ Fottawa citizen.com $\% 2$ Fnews $\%$ 2Flocal-news $\% 2$ Fsix-more-opioid-overdose-victims -treated-at-the-ottawa-hospital

7. Porta $M$ (dir.). A dictionary of epidemiology (6th ed.). International Epidemiological Association. New York (NY) : Oxford University Press; 2014.
8. Institut canadien d'information sur la santé (ICIS). Préjudices liés aux opioïdes au Canada. Ottawa (Ontario) : ICIS; 2017. 